INRA Prod. Anim., 2004, 17 (3), 199-204

\section{A. FOSTIER, B. JALABERT}

INRA, Station Commune de Recherche en Ichtyophysiologie, Biodiversité et Environnement, Campus de Beaulieu, F-35042 Rennes Cedex

Courriel :

fostier@beaulieu.rennes.inra.fr

\title{
Domestication et reproduction chez les poissons
}

La grande diversité et la richesse des stratégies de reproduction chez les poissons donnent un intérêt particulier à la question de la maîtrise de la reproduction d'une nouvelle espèce d'élevage au cours de son processus de domestication. Mieux comprendre ce processus et rationaliser davantage le choix des méthodes de maîtrise de la reproduction sont des enjeux importants d'un point de vue économique ou zootechnique, mais aussi scientifique. Les démarches de recherche et développement devraient pouvoir évoluer en s'appuyant sur l'essor d'une biologie comparée bénéficiant des nouveaux acquis sur la connaissance des génomes et de l'évolution des méthodes de traitement des bases de données.

L'exigence de maîtrise de la reproduction de nouvelles espèces peut être plus ou moins forte selon le système d'élevage choisi et les contraintes économiques qu'il doit intégrer. On considère, en général, que des systèmes intensifs sont plus exigeants que des sys-

\section{Résumé}

La demande de diversification des produits piscicoles conduit à mettre en élevage et domestiquer de nouvelles espèces de poissons. Une des étapes de cette domestication vise à maîtriser la reproduction, à un niveau de sophistication qui peut d'ailleurs varier selon le degré d'intensification du système d'élevage. Une réflexion sur la rationalisation de cette démarche conduit à explorer trois types de questions : Quelle maîtrise de la reproduction pour les espèces d'élevage actuelles? Comment extrapoler les connaissances acquises d'une espèce à une autre ? Quelles sont les caractéristiques de reproduction les plus intéressantes à prendre en compte dans le processus de sélectiondomestication?

La réponse à la première question montre des niveaux de contrôle de la reproduction très variables selon les espèces. Ceci tient à la diversité de l'état des connaissances, des disponibilités en juvéniles sauvages et des choix techniques aujourd'hui économiquement viables.

La seconde question soulève le problème des classifications et typologies génétiques et phénotypiques disponibles et de leur exploitation. Les bases de données qui se développent permettent de proposer des phylogénies s'appuyant sur la connaissance du génome et de son expression, mais le couplage avec des bases de données, aussi riches et codifiées, portant sur les caractères phénotypiques des espèces considérées reste encore un enjeu dans une démarche in silico de biologie comparée.

Dans un objectif utilitaire, la réponse à la troisième question peut guider les choix à faire parmi les différents traits caractérisant une stratégie de reproduction, ceci pour orienter la recherche de certaines convergences biologiques et les extrapolations de techniques d'une espèce à une autre. tèmes extensifs. Ainsi, une écloserie devra utiliser au mieux son potentiel de production en contrôlant, tout au long de l'année, les périodes et les quantités d'alevins à produire en adéquation avec son marché. Par ailleurs, l'obtention de reproductions satisfaisantes dans des systèmes extensifs au fonctionnement complexe (étangs par exemple) peut également nécessiter une connaissance élaborée de la reproduction des espèces concernées, afin d'intervenir de manière rationnelle sur des facteurs dont les effets biologiques résultent souvent d'interactions (qualité de l'eau, température, photopériode, facteurs trophiques, facteurs sociaux ...). Enfin, même dans des systèmes où la reproduction échappe au contrôle direct de l'éleveur, celui-ci est susceptible d'exercer une pression de sélection indirecte à travers ses pratiques d'élevage.

Une plus grande maîtrise de la production dans les filières en émergence ou la volonté de diversifier les produits par l'offre de nouvelles espèces conduisent à une demande récurrente d'un contrôle optimal de la reproduction. Quel est l'état de cette maîtrise selon les espèces d'élevage actuelles ? Peut-on, et comment, extrapoler les connaissances acquises d'une espèce à une autre ? Parmi ces connaissances, quelles peuvent être les caractéristiques de reproduction les plus intéressantes à prendre en compte dans le processus de sélection-domestication? Le texte qui suit s'arrête sur ces questions. 


\section{1 / Des niveaux divers de maîtrise de la reproduction chez les poissons d'élevage}

Le processus de domestication est un phénomène progressif qui peut être défini comme une co-évolution entre certaines caractéristiques de l'espèce, dont les modalités de reproduction, et les rapports de l'homme avec cette espèce à travers ses pratiques d'élevage. Alors que ce processus s'est déroulé depuis des millénaires pour diverses espèces domestiques de vertébrés supérieurs, le phénomène est beaucoup plus récent pour les poissons, chez lesquels on peut distinguer quatre grands groupes d'espèces.

Des espèces dont la reproduction en captivité est maîtrisée de plus ou moins longue date et pour lesquelles on peut effectivement admettre l'existence d'une co-évolution avec des pratiques d'élevage comportant éventuellement une démarche de sélection génétique, plus ou moins empirique au début, et maintenant de plus en plus rationalisée. Il s'agit notamment de plusieurs espèces appartenant à la famille des cyprinidés (carpes) et des salmonidés (truites et saumons) dont la production mondiale en élevage avoisinait respectivement 14,8 et 2,7 millions de tonnes en 2002 (FAO 2004).

Des espèces dont la reproduction en captivité a été maîtrisée récemment, pour lesquelles la co-évolution avec les systèmes d'élevage ne fait que débuter, mais qui font l'objet, pour plusieurs d'entre elles, de programmes d'amélioration génétique. La liste de ces espèces s'allonge en permanence : bar, daurades, turbot, poissons-chats africain (Clarias), américain (Ictalurus punctatus), asiatique (genre Pangasius), milkfish (Chanos chanos), morue, sériole, ombrine tropicale (Sciaenops ocellatus), barramundi (Lates calcarifer), mérous ...

Des espèces comme l'anguille européenne, dont la reproduction en élevage n'a jamais été obtenue, mais donnant lieu à une production aquacole.

Des espèces comme les tilapias (notamment du genre Oreochromis) dont la reproduction spontanée en élevage, paradoxalement, pose problème du fait de l'excessive prolifération des alevins.

\section{2 / Des approches compara- tives pour transférer des connaissances d'une espèce à une autre}

Pour maîtriser la reproduction d'une nouvelle espèce, on sera évidemment tenté d'appliquer les méthodes ayant fait leurs preuves sur les espèces domestiquées. Faisant cela, on extrapole, parfois plus ou moins consciemment, les connaissances déjà acquises sur d'autres espèces. Au-delà de l'in- tuition, existe-t-il des méthodes rationnelles permettant d'extrapoler les connaissances de la biologie de la reproduction d'une espèce à une autre?

\section{1 / Utilisation des classifications d'espèces}

L'hypothèse forte qui sera faite dans l'utilisation de classifications d'espèces sera celle d'une similarité des biologies de la reproduction d'autant plus grande que les espèces seront proches dans l'ordonnancement (ou proches dans les ramifications des arbres phylogénétiques). Cette hypothèse est couramment faite dans les extrapolations réalisées au sein d'un genre ou d'une famille. La validité de cette hypothèse dépendra en particulier de la nature des critères de classification. La prise en compte de données sur la biologie de la reproduction, comme par exemple dans le groupe très diversifié des tilapias (Trewavas 1982), sera évidemment favorable.

D'après une base de données couramment utilisée par les ichtyologistes (Fishbase : www.fishbase.org), une classification générale en taxinomie vise à 'un arrangement des organismes dans une hiérarchie nomenclaturale, selon leur degré évolutionnaire'. En fait, il existe différentes classifications dont les propriétés font l'objet de débats. L'une des questions, qui nous intéresse particulièrement ici, porte sur la relation entre les fonctions et les critères utilisés, basés sur des travaux de paléontologie, de morphologie, de biochimie, d'immunologie, sur l'étude des allozymes-isozymes, des séquence d'acides aminés, de l'ADN mitochondrial, de l'ADN nucléaire ... (Nelson 1987). Les phylogénies peuvent en effet être basées sur des critères phénotypiques, surtout morphologiques, ou reposer sur une évaluation de distance génétique à l'aide de marqueurs moléculaires polymorphes et ubiquistes. Dans ce second cas, la question du lien avec les fonctions est formulée comme celle du lien entre variabilité génétique des marqueurs retenus et variabilité des locus des caractères d'intérêt (Ollivier et al 2000). Quoi qu'il en soit, les phylogénies basées sur des critères morphologiques ne sont pas toujours cohérentes entre elles. Elles peuvent aussi différer des classifications basées sur des critères moléculaires (Inoue et al 2003), ces dernières pouvant chercher à intégrer des données recueillies sur l'ADN aussi bien mitochondrial que nucléaire (Crespi et Fulton 2004). Des différences peuvent également provenir du choix de la méthode d'analyse des données (Reed et al 2002).

Par construction, les phylogénies permettent de s'appuyer sur des similarités évolutives. Se posera cependant la question du niveau hiérarchique à considérer en fonction du caractère biologique étudié, qui doit bien entendu se situer après un point de divergence pour ce caractère. Par exemple, au sein de l'ordre des anguilliformes, les familles des anguillidaes (anguille européenne, anguille japonaise) et des congridaes (congres) pré- 
sentent des ovogenèses similaires (Utoh et al 2003), mais diffèrent considérablement pour d'autres caractéristiques de leur reproduction. Par ailleurs, on trouvera au sein de la famille des sparidés (ordre des perciformes) une grande diversité dans la sexualité : si l'on s'intéresse à une nouvelle espèce de sparidé d'intérêt aquacole (Fostier et al 2000), on pourra s'attendre à l'éventualité d'un hermaphrodisme (information apportée par la connaissance d'un certain nombre d'espèces de ce groupe de la systématique des poissons), mais sans pouvoir conclure sur la nature réelle de la sexualité de l'espèce considérée qui pourra être protandre, protogyne ou gonochorique (Devlin et Nagahama 2002 ). D’un autre côté, l'information sur la diversité des sexualités au sein de l'ordre des perciformes peut laisser prévoir un déterminisme génétique faible du sexe chez une espèce réputée gonochorique comme le bar (Saillant et al 2003).

\section{2 / L'hypothèse de régulations conservées}

Une hypothèse fréquente, mais pas toujours explicite (ni même toujours consciente !), faite pour extrapoler des données acquise d'une espèce à une autre est celle de la conservation, au cours de l'évolution, d'une régulation particulière. Il s'agit parfois d'une intuition, mais ceci résulte plutôt de l'accumulation de données similaires pour des espèces phylogénétiquement éloignées, pouvant être renforcée par le fait que la régulation de l'élément de fonction étudié est considéré comme majeur et que, par ailleurs, les facteurs de régulation sont déjà connus comme relativement universels (certains peptides ou stéroïdes par exemple). Ceci est souvent vérifié pour des régulation intra-cellulaires, comme dans le cas du MPF, facteur ovocytaire induisant la reprise de méiose chez les poissons (Jalabert et al 1991). Ce peut être vrai aussi pour des régulations à l'échelle de l'organisme comme, par exemple, la régulation de la vitellogenèse par une gonadotropine (plutôt FSH chez les salmonidés) stimulant la synthèse d'oestradiol ovarien qui lui-même va stimuler la synthèse de vitellogénine hépatique. Ce modèle de régulation de la vitellogenèse est devenu quasiment universel et les données les plus récentes sur de nouvelles espèces ne dérogent pas à la règle (par exemple, pour le hareng du Pacifique : Koya et al 2003).

Une stratégie d'exploitation plus rationnelle de cette démarche pourrait être, pour une régulation particulière, d'une part de choisir quelques modèles phylogénétiquement éloignés pour mieux tester l'hypothèse avant de la généraliser, d'autre part d'établir une typologie des variantes à un modèle principal de régulation à partir d'espèces choisies pour leurs différences phénotypiques remarquables par rapport à la fonction (ou l'élément de fonction) étudiée. Ainsi, dans le cas de la régulation de la vitellogenèse, il serait sans doute utile de mieux comprendre les différences existantes selon les types d'ovogenèse connus, en particulier entre les ovogenèses à développement synchrone et asynchrone des ovocytes.

Il faut bien entendu prendre garde aux dérives dogmatiques de l'utilisation de tels modèles universels. Pour rester sur l'exemple de la vitellogenèse, l'importance simplificatrice donnée à l'oestradiol peut ainsi masquer les actions plus discrètes, mais importantes, d'autres oestrogènes (van Bohemen et al 1982). Par ailleurs, les rôles respectifs de LH et FSH dans la régulation des niveaux sanguins de l'œestradiol méritent d'être encore explorés (Gen et al 2000).

\section{3 / Recherche de gènes orthologues et analyse du degré d'homologie}

On peut aussi considérer comme favorable à une extrapolation l'existence d'homologies de séquences en se focalisant sur un gène ou sur un groupe de gènes, donc sur une régulation ou un phénomène particulier. Ainsi, l'analyse phylogénétique des gènes codant pour les gonadotropines a permis de confirmer clairement l'homologie des deux gonadotropines de poisson (initialement dénommées GTH-1 et GTH-2) aux LH et FSH des tétrapodes (Quérat et al 2000). La détection, chez une nouvelle espèce, de gènes orthologues d'espèces connues permet aussi de faire l'hypothèse de l'existence de fonctions comparables. Un exemple récent concerne l'identification d'un gène de récepteur membranaire aux progestines impliqué dans le contrôle de la maturation méiotique qui laisse penser à une généralisation de ce mécanisme d'action (Zhu et al 2003). Enfin, la reconnaissance de différents variants présents ou non chez les espèces considérées doit aussi permettre de conclure sur l'existence ou non de certains types de régulation, comme dans le cas des variants de la gonadolibérine ( 8 formes de $\mathrm{GnRH}$ chez les téléostéens) et de ses récepteurs (Lethimonier et al 2004).

\section{4 / Des convergences biologiques ayant des conséquences zootechniques}

D'une manière plus empirique, on peut tenter de transposer des techniques en ne considérant que les convergences phénotypiques. C'est probablement la démarche la plus fréquente dans les premières étapes de la domestication en complément de la prise en compte des données disponibles sur l'écobiologie de l'espèce sauvage mise en élevage. Relèvent souvent de cette démarche : l'induction de ponte (gonadolibérine considérée comme régulateur universel : Mylonas et Zohar 2001), les conditions d'une fécondation in vitro (généralisation au sein des ovipares), le choix d'un mode de traitement et d'incubation des œufs selon leur type (œufs pélagiques, adhésifs ou benthiques). La domestication d'une espèce peut commencer par l'obtention de pontes spontanées après acclimatation de quelques géniteurs en bassin, ou bien par une identification succincte de sa gamétogenèse et l'ajustement de proto- 
coles d'induction de ponte puis de fécondation in vitro (Hassin et al 1997). Ce sera, de fait, une première étape de sélection d'individus adaptés aux techniques choisies (pression de sélection sur les géniteurs, les œufs, les alevins).

\section{5 / Perspectives}

L'essor de la génomique et l'enrichissement des bases de données sur les séquences et la fonctionnalité des gènes encouragent deux approches complémentaires utiles à la biologie comparée. D’une part, la construction de modèles potentiellement généralisables par l'identification de gènes (ou mieux, de réseaux de gènes) fortement conservés ; d'autre part, la recherche, chez une nouvelle espèce, de l'existence de gènes homologues à ceux déjà reconnus comme importants dans une fonction particulière.

Cependant, par rapport à la question initiale posée (comment généraliser la connaissance de la biologie de la reproduction d'une espèce à une autre ?), on peut penser que l'information la plus utile ne sera pas la présence de tels ou tels gènes homologues, mais plutôt l'existence de profils d'expression comparables de ces gènes dans des situations physiologiques données (situations choisies comme clefs dans une fonction particulière de la reproduction). Ceci nécessite un choix pertinent du groupe de gènes à étudier et des situations physiologiques retenues, ainsi que des outils d'analyse d'expression 'à haut débit', c'est-à-dire, pour ce dernier point, une bonne maîtrise du travail avec des outils hétérologues si l'on veut effectivement bénéficier des acquis obtenus sur une espèce 'modèle'

Les outils d'analyse d'expression 'à haut débit' concernent aujourd'hui majoritairement l'analyse des transcrits des gènes (transcriptome), ce qui n'est qu'une étape de leur expression fonctionnelle. Le développement d'analyses à 'haut débit' pour les protéines (protéome) et les métabolites formés (métabolome) doit compléter cette information.

Il est encore difficile de croire que de telles analyses systématiques pourront être entreprises sur de nombreuses espèces. C'est pourquoi le choix des espèces modèles de référence est particulièrement important et ce choix renvoie aux classifications sur lesquelles il peut reposer de manière rationnelle. Doit-on alors s'appuyer sur une classification strictement phylogénétique davantage résolutive (Miya et al 2003), ou également prendre en compte une classification phénotypique (prise en compte des convergences fonctionnelles) ? Il serait alors sans doute utile de disposer de bases de données codifiées pour les caractères phénotypiques permettant de traiter in silico un grand nombre d'informations. Des typologies ont été proposées, le plus souvent dans un contexte écologique, pour des traits caractérisant des stratégies de reproduction (Balon 1975, Wootton 1999, Zeller et Pauly 2001). Parfois elles visent un phénomène particulier, comme la vitellogenèse (Wallace et Selman 1981), et prennent en compte des régulations physiologiques. Des bases de données phénotypiques pourraient être alimentées par ce type de travaux.

\section{3 / Caractéristiques de la reproduction pouvant être prises en compte dans un processus de sélection-domestication}

Les diverses espèces de poissons présentent une très grande variété de stratégies et tactiques de reproduction (Wootton 1984). Classiquement, la notion de "stratégie de reproduction " désigne un ensemble de caractéristiques propres à une espèce considérée, déterminé par son génome, alors que le terme " tactiques de reproduction " désigne diverses adaptations phénotypiques résultant de la réponse de ce génome à des environnements variés, du fait d'une certaine plasticité physiologique et comportementale. Pratiquement, la possibilité d'obtenir d'emblée la reproduction en captivité d'une espèce a priori sauvage va dépendre, d'une part, de sa stratégie de reproduction et, d'autre part, de sa capacité à ajuster sa tactique de reproduction aux conditions offertes par le milieu d'élevage. Ainsi, la stratégie de reproduction de l'anguille européenne (un seul cycle reproducteur au terme d'une longue migration océanique accompagnée de profondes transformations physiologiques) s'avère très défavorable, alors que la stratégie de reproduction d'espèces comme les tilapias ou de nombreux cyprinidés, dont le cycle reproducteur peut se dérouler entièrement dans un même milieu relativement fermé, est beaucoup plus favorable. Au-delà de la plasticité phénotypique des tactiques de reproduction d'une espèce, c'est la variabilité d'origine génétique de sa stratégie de reproduction qui peut, en donnant prise à une sélection dans le milieu d'élevage, permettre une évolution vers la domestication. Cet effort de sélection génétique peut concerner plusieurs caractéristiques.

Ainsi, la possibilité de maîtriser différenciation ou inversion sexuelle chez les poissons est un atout souvent recherché dans les élevages de poissons (Guiguen et al 1996). Chez les espèces hermaphrodites comme le barramundi (espèce protandre, Guiguen et al 1994) ou les mérous (protogynes, Debas et al 1989), il peut être avantageux d'avancer ou de retarder, par sélection, le moment de l'inversion sexuelle. Chez certaines espèces gonochoriques, comme les tilapias ou le bar, la différenciation sexuelle peut s'avérer sensible à l'action de facteurs externes comme la température (Baroiller et D'Cotta 2001) et il est envisageable de sélectionner des souches particulièrement sensibles à ces facteurs, afin de les utiliser pour orienter la sex-ratio en élevage dans le sens le plus favorable à l'objectif de production.

Lâge et/ou la taille à la première reproduction est un paramètre important qui peut affecter plus ou moins directement la rentabilité de l'élevage selon les espèces et les objectifs de production. En général, dans la 
mesure où l'élaboration des gonades s'effectue au détriment de la croissance, la précocité excessive est un inconvénient majeur, comme dans le cas des tilapias où cette précocité s'accompagne en outre d'une prolifération d'alevins incontrôlée. A l'inverse, on peut toutefois citer le cas extrême de l'esturgeon, dont la puberté tardive (au-delà de 6 à 7 ans chez l'esturgeon sibérien élevé en France) est un inconvénient pour la production de caviar. Ce caractère de la stratégie de reproduction des espèces présente donc généralement un très fort déterminisme génétique qui peut être toutefois fortement influencé par les facteurs externes qui modulent la croissance, en particulier la température et l'alimentation.

Le cycle reproducteur représente une contrainte forte dans l'organisation des élevages. C'est en fait la succession des phénomènes physiologiques qui se déroulent entre deux périodes de frai et se manifestent par le développement progressif des gonades (spermatogenèse et vitellogenèse) jusqu'à l'évacuation des gamètes (spermiation et ovulation). Ce cycle est généralement annuel chez les espèces de zones tempérées, fortement inféodées au rythme saisonnier des facteurs externes. Chez ces espèces, la photopériode est généralement le principal facteur d'entraînement du cycle, les autres facteurs n'intervenant que pour ajuster la ponte au moment le plus favorable (Bromage et al 2001). Si le contrôle photopériodique du cycle reproducteur est fréquemment utilisé dans la pratique piscicole pour ajuster la production des œufs à la demande, la qualité de ces oufs semble toutefois dépendre d'interactions encore mal maîtrisées entre des caractéristiques génétiques mal définies d'une part, et l'action de facteurs externes autres que la photopériode d'autre part, notamment la température. Chez la truite arcen-ciel, il existe ainsi diverses souches caractérisées par des saisons de ponte naturelle différentes et susceptibles de répondre différemment aux variations de photopériode. Chez cette espèce, une souche présentant deux cycles reproducteurs annuels sous photopériode naturelle a d'ailleurs été sélectionnée au Japon (Tazaki et al 1993).

La plasticité des tactiques et la variabilité dans la stratégie de reproduction de certaines espèces ont déjà pu être exploitées, dans une première phase de domestication plus ou moins empirique, pour satisfaire en partie aux contraintes de l'élevage et aux besoins de la production. Mais la plupart des caractères d'intérêt peuvent présenter, selon les espèces, une plus ou moins grande variabilité de leur sensibilité à l'action de facteurs externes tels que la photopériode, la température, la salinité ou le stress. Une seconde phase de domestication pourrait donc certainement s'appuyer sur une meilleure connaissance des modalités de réponse physiologique des diverses espèces à l'action des facteurs externes (seuils et plages de réponse, interactions) en vue de sélectionner les modalités de réponse les mieux adaptées aux objectifs de production.

\section{Conclusion}

Nous avons essentiellement abordé la question de la domestication sous son angle utilitaire, à savoir comment tirer des règles générales et rationaliser davantage des méthodes de domestication pour, plus rapidement et plus efficacement, mettre en élevage de nouvelles espèces d'intérêt économique. L'adaptation des populations d'élevage à leur environnement peut conduire à un certain paradoxe, dans les résultats obtenus, entre une volonté de maîtrise technique élevée ('biotechnologies') et une bonne adaptation des populations. Ainsi au début des années 1970, la ponte de la daurade royale, Sparus auratus, n'était obtenue qu'après induction hormonale, d'où le développement de techniques d'induction de plus en plus efficaces par des gonadolibérines. Aujourd'hui, après plusieurs générations reproduites en captivité, des pontes spontanées sont obtenues dans les bassins (Zohar et Mylonas 2001).

Il est par ailleurs possible d'aborder la question de la domestication en se demandant quel peut être son effet sur les caractéristiques de reproduction. Outre les informations qui peuvent être tirées pour un objectif d'élevage, cette analyse donne des éléments utiles pour répondre à la question des effets potentiels d'échappées de populations domestiques sur l'évolution et le devenir des populations naturelles. La démarche peut être faite a posterio$r i$ en comparant les caractéristiques de populations domestiquées à des populations sauvages, quand elles existent encore ( Petersson et al 1996). Elle pourrait être engagée a priori en suivant l'évolution de ces caractéristiques au cours d'un processus de domestication sur une nouvelle espèce. Ceci peut se faire en associant analyse de l'évolution des phénotypes et analyse de l'évolution des génotypes et de leur fonctionnement.

\section{Références}

Balon E.K., 1975. Reproductive guilds of fishes: a proposal and definition. J. Fish. Res. Board Can., 32, 821-864.

Baroiller J.F., d'Cotta H., 2001. Environment and sex determination in farmed fish. Comp. Biochem. Physiol. C, 130, 399-409.

Bromage N., Porter M., Randall C., 2001. The environmental regulation of maturation in farmed finfish with special reference to the role of photoperiod and melatonin. Aquaculture, 197, 63-98.

Crespi B.J., Fulton M.J., 2004. Molecular systematics of Salmonidae: combined nuclear data yields a robust phylogeny. Mol. Phylogenet. Evol., 31, 658-679.
Debas L., Fostier A., Fuchs J., Wappe M., Nedelec G., Bennett A., Auacop, Cauty C., Jalabert B., 1989. The sexuality of cultured hermaphroditic fish species: analysis of morphological and endocrinological features in a protogynous hermaphrodite Epinephelus microdon as a basis for further research to control reproduction of the grouper. In : Advances in Tropical Aquaculture, Actes de colloques $n^{\circ} 9$, 543-557. Editions Ifremer, Paris.

Devlin R.H., Nagahama Y., 2002. Sex determination and sex differentiation in fish: an overview of genetic, physiological, and environmental influences. Aquaculture, 208, 191-364. 
FAO, 2004. FAO yearbook. Fishery statistics. Aquaculture production 2002. Vol. 94/2.

Fostier A., Kokokiris L., Le Menn F., Mourot B., Pavlidis M., Divanach P., Kentouri M., 2000. Recent advances in reproductional aspects of Pagrus pagrus. Seminar on Mediterranean Marine Aquaculture Finfish Species Diversification. Cah. Options Méditerr., 47, 181-192.

Gen K., Okuzawa K., Senthilumaran B., Tanaka H. Moriyama S., Kagawa H., 2000. Unique expression of gonadotropin-I and -II subunit genes in male and female red seabream (Pagrus major) during sexual maturation. Biol. Reprod., 63, 308-319.

Guigen Y., Cauty C., Fostier A., Fuchs J., Jalabert B., 1994. Reproductive cycle and sex inversion in the sea bass Lates calcarifer reared in sea cages in French Polynesia: histological and morphometric description. Environ. Biol. Fish., 39, 231-247.

Guiguen Y., Baroiller J.F., Jalabert B., Fostier A., 1996. Le contrôle du sexe phénotypique chez les poissons. Pisc Française, 124, 16-19.

Hassin S., de Monbrison D, Hanin Y, Elizur A, Zohar Y, Popper D.M., 1997. Domestication of the white grouper, Epinephelus aenus. 1. Growth and reproduction. Aquaculture, 156, 305-316.

Inoue J.G., Miya M., Katsumi Tsukamoto K, Nishida M. 2003. Basal actinopterygian relationships: a mitogenomic perspective on the phylogeny of the "ancient fish". Mol Phylogenet. Evol., 26, 110-120.

Jalabert B, Fostier A, Breton B, Weil C, 1991. Oocyte maturation in vertebrates. In: P.K.T. Pang and M.P. Schreibman (eds), Vertebrate endocrinology: fundamentals and biomedical implication, Vol 4A, 23-90. Academic Press, N.Y.

Koya Y., Soyano K., Yamamoto K., Obana H., Matsubara T., 2003. Oocyte development and serum profiles of vitellogenin and steroid hormone levels in captive female Pacific herring Clupea pallasii during their first maturationa cycle. Fisheries Sci., 69, 137-145.

Lethimonier C., Madigou T, Munoz-Cueto J.A., Lareyre J.J., Kah O., 2004. Evolutionary aspects of GnRHs, GnRH neuronal systems and GnRH receptors in teleost fish. Gen. Comp. Endocrinol., 135, 1-16.

Miya M. Takeshima H., Endo H., Ishiguro N.B., Inoue J.G., Mukai T., Satoh T.P., Yamaguchi M., Kawaguchi A. Mabuchi K., Shirai S.M., Nishida M., 2003. Major patterns of higher teleostean phylogenies: a new perspective based on 100 complete mitochondrial sequences. Mol. Phylogenet. Evol., 26, 121-138.

Mylonas C., Zohar Y., 2001. Endocrine regulation and artificial induction of oocyte maturation and spermiation in basses of the genus Morone. Aquaculture, 202, 205-220.

Nelson J.S., 1987. The next 25 years: vertebrates systematics. Can. J. Zool., 65, 779-785.

Ollivier L., Chevalet C., Foulley J.L., 2000. Utilisation des marqueurs pour la caractérisation des ressources géné- tiques. INRA Prod. Anim., numéro hors série « Génétique moléculaire : principes et application aux populations animales », 247-252.

Petersson E., Järvi T., Steffner N.G., Ragnarsson B., 1996 The effect of domestication on some life history traits of sea trout and Atlantic salmon. J. Fish. Biol., 48, 776-791.

Quérat B., Sellouk A., Salmon C., 2000. Phylogenetic analysis of the vertebrate glycoprotein hormone family including new sequences of sturgeon (Acipenser baeri) $\beta$ subunits of the two gonadotropins and the thyroid-stimulating hormone. Biol. Reprod., 62, 222-228.

Reed D.L., Carpenter K.E., deGravelle M.J., 2002 Molecular systematics of the Jacks (Perciformes: carangidae) based on mitochondrial cytochrome b sequences using parsimony, likehood, and Bayesian approaches. Mol. Phylogenet. Evol., 23, 513-524.

Saillant E., Chatain B., Menu B., Fauvel C.,Vidal M.O., Fostier A, 2003. Sexual differentiation and juvenile intersexuality in the European sea bass (Dicentrarchus labrax). J. Zool., 260, 53-63.

Tazaki, S., Nomura H., Suzuki K., 1993. Reproduction characteristics of twice-annually spawning strain of rainbow trout, Oncorhynchus mykiss. Bull. Saitama Pref. Fish Exp. Stat., 51, 63-71.

Trewavas E., 1982. Genetic groupings of Tilapiini used in aquaculture. Aquaculture, 27, 79-81.

Utoh T., Horie N., Okamura A., Yamada Y., Tanaka S. Mikawa N., Akazawa A., Poka H.P., 2003. Oogenesis in the common Japanese conger Conger myriaster. Fisheries Sci., 69, 181-188.

Van Bohemen C.G., Lambert J.G.D., van Oordt P.G.W. 1982. Vitellogenin induction by estradiol in estrone-primed rainbow trout, Salmo gairdneri. Gen. Comp. Endocrinol. 46, 136-139.

Wallace R.A., Selman K., 1981. Cellular and dynamic aspects of oocyte growth in teleosts. Amer. Zool., 21, 325-343.

Wootton R.J., 1984. Introduction: tactics and strategies in fish reproduction. In: Fish Reproduction: Strategies and tactics, Poots G.W., Wootton R.J. (eds), Academic Press, London, pp. 1-12.

Wootton R.J., 1999. Reproduction. In: R.J. Wootton (ed) Ecology of Teleost Fishes. Fish and Fisheries Series, vol 24 141-175. Kluwer Academic Publishers, Dordrecht.

Zeller D., Pauly D., 2001. Visualisation of standardized life-history patterns. Fish Fisheries, 2, 344-355.

Zhu Y., Bond J., Thomas P., 2003. Identification, classification, and partial characterization of genes in humans and other vertebrates homologous to a fish membrane progestin receptor. Proc. Natl. Acad. Sci. USA, 94, 6803-6808.

Zohar Y., Mylonas C., 2001. Endocrine manipulations of spawning in cultured fish: from hormones to genes. Aquaculture, 197, 99-136.

\section{Abstract}

\section{Domestication and reproduction in fish.}

Diversification of the seafood market requires to attempt the domestication of new fish species. Reproduction control is more or less advanced depending on farming intensification level. In view of rationalizing methods for the domestication of new species, three questions are discussed : To what extent the reproduction of the species currently farmed is actually controlled? How to extrapolate the knowledge from a well-known species to another? What are the main reproductive features to consider when trying to domesticate new species?

First, the extent of artificially controlled reproduction varies greatly with species. This may be due to more or less advanced biological knowledge, availability of juve- niles from the wild, and economical and technical choices

The second question is dealing with the elaboration, the choice, and the use of species classifications based on genetics characters or on phenotypes. Genetics and genomics data banks are developing but need to be connected with data banks describing judicious reproductive characteristics. The development of relevant typologies for these characteristics and the availability of adequate bioinformatics tools would be useful.

The third point of discussion is focusing on a limited number of teleostean reproductive features into which it could be worth to go further in a zootechnical prospect.

FOSTIER A., JALABERT B., 2004. Domestication et reproduction chez les poissons. INRA Prod. Anim., 17, 199-204. 